\title{
Enxerto dermoadiposo em cavidades anoftálmicas secundárias - estudo retrospectivo e revisão da literatura
}

\author{
Dermofat graft in secondary anophthalmic \\ socket - a retrospective study and literature review
}

Silvia Narikawa ${ }^{1}$, Silvana Artioli Schellini², Carlos Roberto Padovani ${ }^{3}$

\section{Resumo}

Objetivo: Avaliar o uso do enxerto dermoadiposo no tratamento da cavidade anoftálmica. Métodos: Estudo retrospectivo, do qual participaram 28 portadores de cavidade anoftálmica secundária, tratados com enxerto dermoadiposo. Os dados avaliados foram:idade, sexo, motivo da primeira cirurgia, tipo de cavidade, razão da opção pelo enxerto dermoadiposo, resultados e complicações. Os dados foram analisados segundo a frequência de ocorrência. Resultados: dos 28 pacientes analisados, 19 (67,8\%) eram do sexo masculino, 46,4\% deles apresentando de 40 a 60 anos de idade. A principal causa da perda do olho foi o trauma ( 7 casos $-25,0 \%$ ). A cavidade onde mais se empregou o enxerto dermoadiposo foi a do tipo III $(32,1 \%)$. Como primeira cirurgia, a evisceração havia sido realizada em 13 casos, a enucleação em 11 e o enxerto de pele em dois pacientes e em outros dois, não havia informação sobre a primeira cirurgia a que haviam sido submetidos. A opção pelo enxerto dermoadiposo foi feita com a finalidade de repor volume na cavidade e, ao mesmo tempo, conseguir maior superfície anterior da cavidade, já que a maioria possuía cavidades contraídas. A maioria dos pacientes $(53,6 \%)$ não apresentou complicações, tendo sido a necrose do enxerto a complicação mais observada $(32,1 \%)$. O resultado final da cirurgia foi considerado bom em $71,4 \%$ dos pacientes e ruim em 7,1\%. Conclusão: O enxerto dermoadiposo é uma técnica cirúrgica simples e de bons resultados para reposição de volume em cavidades anoftálmicas. Os autores estimulam o ensino da mesma nos serviços de treinamento de jovens cirurgiões.

Descritores: Anoftalmia/cirurgia; Implantes orbitários/métodos; Procedimentos cirúrgicos reconstrutivos; Tecido adiposo/transplante

\footnotetext{
'Professora Assistente do Departamento de OFT/ORL/CCP, Faculdade de Medicina de Botucatu da Universidade Estadual "Júlio de Mesquita Filho" - UNESP - Botucatu (SP), Brasil;

${ }^{2}$ Professora Titular do Departamento de OFT/ORL/CCP, Faculdade de Medicina de Botucatu da Universidade Estadual "Júlio de Mesquita Filho" - UNESP - Botucatu (SP), Brasil;

3 Professor Titular do Departamento de Bioestatística, Instituto de Biociências da Universidade Estadual "Júlio de Mesquita Filho" UNESP - Botucatu (SP), Brasil.

Trabalho realizado na Faculdade de Medicina de Botucatu - Universidade Estadual "Júlio de Mesquita Filho" - UNESP - Botucatu (SP), Brasil.
}

Os autores declaram inexistir conflitos de interesse

Recebido para publicação em: 8/12/2010 - Aceito para publicação em 2/6/2011 


\begin{abstract}
Purpose: To evaluate the dermofat graft in the anophthalmic socket treatment. Methods: A retrospective study including 28 anophthalmic cavity carriers treated with dermofat graft was carried out. The patients were analyzed according to age, sex, socket contraction degree, first surgery performed, reason for graft choice, procedure's results and complications. The data were analyzed according to the frequency of occurrence. Results: Nineteen $(67.8 \%)$ of the 28 analyzed patients were male. According to age the majority of patients were between 40 to 60 years old (13 patients $-46.4 \%$ ) and the most common cause of eyeball loss was trauma (seven cases $-25 \%$ ). Anophthalmic socket type III was the most frequently observed (32.1\%). The evisceration was the first procedure in 13 cases, enucleation in 11 and skin graft was done in two patients. Two patients came to the service after evisceration and did not provide any information about the first surgery. The choice to use the dermofat graft was to replace volume at the socket of 12 patients (42.8\%). Most of the patients (53.6\%) had no complications and graft necrosis was the problem most frequently observed (32.1\%). The surgery was considered good in $71.4 \%$ and not good in only $7.1 \%$. Conclusion: the dermofat graft is an efficient and easy technique to reconstruct the anophthalmic cavity. The authors suggest this procedure must be introduced in the medical training services since it provide good results.

Keywords: Anophthalmos/surgery; Orbital implants/methods; Reconstructive surgery procedures; Adipose tissue/transplantation
\end{abstract}

\section{INTRODUÇÃO}

$\mathbf{A}$ cavidade anoftálmica é uma realidade presente nos serviços especializados em plástica ocular $^{(1)}$.

A cirurgia de enucleação ou evisceração altera a anatomia e a fisiologia da órbita, levando a deformidades em consequência da perda de volume, com mobilização dos tecidos da cavidade orbitária, assim como a contração dos músculos extraoculares. Estas alterações compõem o quadro clínico da síndrome da cavidade anoftálmica, interferindo, inclusive com a posição da pálpebra ${ }^{(2)}$.

A cavidade anoftálmica pode ser classificada segundo o grau de contração da mucosa conjuntival e dos fundos de saco em: grau I quando não há contração e todos os fundos de saco são normais; grau II quando o fundo de saco inferior é raso; grau III, os fundos de saco inferior e superior são rasos; grau IV, todos os fundos de saco são rasos e grau $\mathrm{V}$, quando existe a microorbitia ${ }^{(1,2)}$.

A reconstrução da cavidade anoftálmica leva em consideração diversos fatores como grau de contração da mucosa conjuntival, intensidade da falta de volume na órbita, se a reparação é primária ou secundária, entre outros ${ }^{(2)}$.

É importante que a reconstrução primária seja sempre priorizada para que se possa obter melhor resultado. A devolução de volume para a cavidade pode ser feita por meio de implantes aloplásticos de diversos formatos, integráveis ou não, compostos de diversos materiais. Materiais autólogos também têm sido sugeridos para este fim, como por exemplo, o enxerto dermoadiposo.
Há muito tempo o enxerto dermoadiposo tem sido utilizado para a reconstrução de cavidades anoftálmicas primárias ou como tratamento secundário, após extrusão ou migração do implante ou no tratamento de cavidades contraídas e enoftalmo ${ }^{(3-8)}$.

O enxerto dermoadiposo é considerado um meio efetivo para repor volume orbitário permitindo mobilidade da prótese ocular, com baixa morbidade e resultado cosmético satisfatório ${ }^{(3,5)}$, sendo a única opção para o tratamento de órbitas complicadas e cavidades contraídas ${ }^{(9)}$.

Além das vantagens que existem na utilização de tecidos autólogos, como a não necessidade de triagem para avaliar possível transmissão de doenças infecciosas e a dispensa os meios de conservação e transporte especiais, ainda existem vantagens adicionais, como a não possibilidade de extrusão ou migração do implante (como pode ocorrer com as esferas aloplásticas), dispensa o uso de material heterólogo para envoltório de implantes (como a esclera), com possibilidade de resultados cosméticos e funcionais muito bons ${ }^{(4,9)}$.

Como desvantagens do método apontam-se a necessidade de se trabalhar em dois sítios (um doador e um receptor), o que torna a cirurgia mais extensa, além da redução gradativa do volume implantado ao longo do tempo.

Um estudo comparando a mobilidade da prótese externa sobre o enxerto dermoadiposo ou implante de esferas sintéticas, mostrou mesma mobilidade nos dois grupos, embora o grupo com enxerto dermoadiposo primário tenha fornecido maior movimento e fundos de saco mais amplos ${ }^{(10,11)}$.

Outro estudo no qual foram avaliados 118 enxer- 
tos dermoadiposos, dos quais 51 primários e realizados após enucleação, 19 após migração de implantes prévios, 19 para correção de deformidades do sulco palpebral superior e nove para expansão de cavidades contraídas, mostrou que o enxerto dermoadiposo foi efetivo para a manutenção do volume orbitário, conservando, ainda, os fundos de saco conjuntivais ${ }^{(12)}$.

Embora a atrofia do enxerto não ocorra com muita frequência, este fenômeno é mais comum nos secundários ${ }^{(12.13)}$, principalmente quando houve danos químicos ou outras causas que resultem maior contração da cavidade. $O$ único caso de atrofia importante de enxerto primário foi notado 30 meses após o procedimento ${ }^{(12)}$. Atrofia do enxerto primário ocorre em menos de 10\% dos casos e em secundários, em menos de $30 \%{ }^{(11)}$.

Para outros, o enxerto dermoadiposo autólogo sobrevive melhor, com menor perda de volume, quando implantado dentro da cápsula de Tenon ${ }^{(14)}$, imediatamente após a remoção do bulbo ocular, com a anastomose dos músculos retos no enxerto ${ }^{(13)}$ e quando o diâmetro anterior do enxerto não ultrapassa $22 \mathrm{~mm}$. Além da sutura dos músculos retos, a sobrevivência do enxerto depende também de sua vascularização precoce e da qualidade da cavidade ${ }^{(6)}$.

Estudo envolvendo 60 cavidades, nas quais foi utilizado enxerto dermoadiposo, mostrou como complicações do procedimento: sete pacientes com falha no recobrimento conjuntival com desenvolvimento de ulceração central, 10 casos com enoftalmo, dois com secreção crônica e descamação, três com granuloma conjuntival, um com infecção do enxerto e um com hematoma do sítio doador. A maioria das complicações ocorreram em cavidades severamente traumatizadas ou quando os portadores de cavidades apresentavam doença sistêmica ${ }^{(15)}$.

Complicações como granulomas piogênicos e hirsutismo do enxerto podem ser facilmente controladas ${ }^{(13)}$.

Desta forma, fica claro que a literatura aponta muitas vantagens para o uso do enxerto dermoadiposo na reconstrução da cavidade anoftálmica, assim como a possibilidade de se ter algumas complicações.

O presente estudo foi desenvolvido com o objetivo de avaliar os casos de cavidade anoftálmica nos quais foi realizado enxerto dermoadiposo como procedimento secundário, a fim de verificar como foi a resposta ao emprego desta técnica.

\section{MÉTOdos}

Estudo retrospectivo, realizado na Faculdade de Medicina de Botucatu - UNESP, São Paulo. Foram avaliados 28 portadores de cavidade anoftálmica secundária, nos quais foi empregada a técnica de enxerto dermoadiposo. Os indivíduos foram operados no período de 2001 a 2006, com seguimento mínimo de seis meses. Os pacientes foram avaliados segundo idade, sexo, tipo de cavidade, primeira cirurgia realizada, causa da opção pelo enxerto, resultado do procedimento e complicações. Os resultados foram avaliados com exame clínico dos pacientes.

Todos os pacientes foram operados segundo a mesma técnica operatória: remoção do enxerto da região glútea usando bisturi e tesoura, após ter sido feita a remoção das camadas superficiais da pele em profundidade estimada pela observação de gotículas de sangue, sinal de se ter atingido a derme. $\mathrm{O}$ tamanho do enxerto foi variável, de acordo com a necessidade do paciente. $\mathrm{Na}$ cavidade orbitária foi feita a abertura da conjuntiva e Tenon com tesoura, criando-se um espaço onde seria colocado o enxerto usando tesoura de Metsembaum. Em seguida, o enxerto era suturado com a Tenon e a conjuntiva usando fio inabsorvível trançado 6-0 (Mersilene, Ethicon), em pontos contínuos.

Os dados foram obtidos a partir da análise dos atendimentos ambulatoriais no serviço de Plástica Ocular e descrições cirúrgicas, ambos contidos no prontuário de cada paciente. Dois dos autores acompanharam os atendimentos de todos os indivíduos incluídos no estudo. A análise dos resultados foi feita usando-se técnica da frequência de ocorrência.

\section{Resultados}

Dos 28 pacientes analisados, $19(67,8 \%)$ eram do sexo masculino e nove $(32,1 \%)$ do sexo feminino.

A faixa etária de maior frequência de ocorrência foi a de 40 a 60 anos (13 pacientes - 46,4\%), seguida por idade maior que 60 anos (nove pacientes), entre 20 - 40 anos (quatro casos) e apenas um caso com idade inferior a 20 anos (Gráfico 1).

Quanto à causa da perda do bulbo ocular, o trauma foi responsável por sete casos $(25,0 \%)$, seguido pelo glaucoma absoluto e phthisis bulbi, com cinco casos cada $(17,8 \%)$, infecção e tumor com três casos cada $(10,7 \%)$ e um paciente com anoftalmia congênita. A causa da perda do bulbo não foi relatada em quatro pacientes $(14,3 \%)$ (Gráfico 2).

A avaliação da cavidade anoftálmica mostrou que nove casos $(32,1 \%)$ eram do tipo III, três $(10,7 \%)$ do tipo IV, três $(10,7 \%)$ do tipo II e apenas dois $(7,1 \%)$ do tipo I. Os demais pacientes analisados não apresentavam o registro deste dado no prontuário.

O enxerto dermoadiposo foi indicado como técnica 


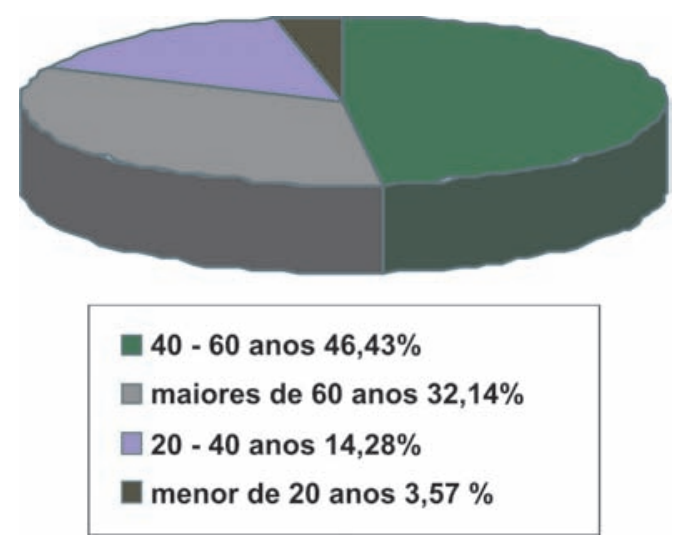

Gráfico 1: Distribuição dos portadores de cavidade anoftálmica tratados com enxerto dermoadiposo segundo faixa etária UNESP, 2010
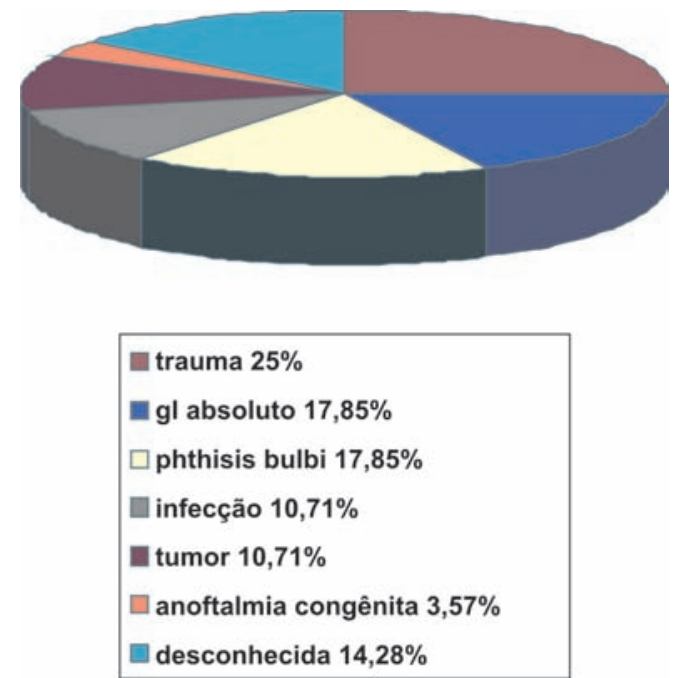

Gráfico 2: Distribuição dos portadores de cavidade anoftálmica que foram tratados com enxerto dermoadiposo segundo as causas da perda do bulbo ocular - UNESP, 2010

a ser executada como segundo procedimento em todos os pacientes, os quais haviam sido submetidos primariamente a evisceração em 13 casos, contra 11 casos de enucleação e dois de enxerto de pele. Em dois casos o paciente chegou ao serviço após a remoção do bulbo ocular, sem informações sobre a primeira cirurgia a que havia sido submetido.

A opção pela cirurgia de enxerto dermoadiposo foi feita por falta de outros meios para repor volume na cavidade de 12 pacientes $(42,8 \%)$. Os demais tiveram a indicação por cavidade contraída (sete casos - 25,0\%), extrusão da prótese (sete casos - 25,0\%) ou deiscência de sutura (dois casos $-7,1 \%$ ).
Quanto às complicações, a maioria dos pacientes $(53,6 \%)$ evoluiu sem nenhuma complicação. Dos demais, nove $(32,1 \%)$ apresentaram necrose do enxerto, quatro $(14,3 \%)$ tiveram deiscência da sutura e dois $(7,1 \%)$ evoluíram com granuloma conjuntival no pós-operatório.

A avaliação dos pacientes foi feita por período mínimo de seis meses. Neste período, avaliando-se subjetivamente os pacientes, o volume do enxerto parecia mantido.

O resultado final da cirurgia foi considerado bom em $20(71,4 \%)$ pacientes e ruim, em apenas dois $(7,1 \%)$. Seis pacientes perderam o seguimento, não tendo sido avaliados neste quesito.

\section{Discussão}

O enxerto dermoadiposo é uma técnica que é usada há muitos anos com o intuito de devolver volume para a cavidade anoftálmica. Em qualquer estudo que envolva cavidade anoftálmica, o sexo masculino será sempre o predominante, em decorrência da atividade laborativa, além da maior exposição a trauma por acidentes em esportes e recreação. O mesmo comentário é válido para faixa etária, quando a faixa etária de adultos jovens sempre será a mais acometida, seguida pelos idosos, os quais estão expostos também a trauma e cirurgias oculares para o tratamento de alterações involucionais. Da mesma forma, o trauma será a causa mais frequente de perda do bulbo na maioria das casuísticas, ou seja, a população acometida reflete o perfil de pessoas expostas e que evoluem com perda do órgão da visão.

Com relação ao tipo de cavidade que levou a indicação do enxerto dermoadiposo, é conduta do serviço usar o enxerto dermoadiposo quando as cavidades possuem contração da mucosa conjuntival, condição na qual a colocação de implantes aloplásticos ou próteses externas não é possível em decorrência da contração dos fundos de saco.

É relevante considerar que não é rotina no serviço suturar os músculos retos ao enxerto e, mesmo assim, a sobrevivência do enxerto é grande. Entretanto, a literatura $^{(10,14)}$ aponta para taxas maiores de sucesso quando se sutura os músculos extrínsecos ao enxerto dermoadiposo por maior irrigação para o enxerto e também pela possibilidade de ter melhor transmissão de movimento dos músculos para a prótese externa.

Apesar de a indicação da técnica ter sido feita mais frequentemente por se tratar de cavidades contraídas, quando a técnica é utilizada para devolução de volume, ao mesmo tempo que aumenta a superfície ante- 
rior da cavidade e facilita a adaptação da prótese externa, em número expressivo de casos foi optado pelo enxerto dermoadiposo por inexistência de esferas aloplásticas no mercado. Este é um fato que está se tornando frequente nos últimos anos, principalmente devido às normas regulatórias da Anvisa, deixando os hospitais sem opção de materiais a preços aceitáveis para tratamento das cavidades anoftálmicas de usuários do Sistema Único de Saúde, que é o caso de nossa clientela.

Quanto às complicações, $32,1 \%$ dos pacientes tiveram necrose do enxerto de diferentes graus de intensidade. Este problema pode decorrer de má técnica operatória (manipulação excessiva do enxerto), presença de infecções que podem estar presentes e inclusive causando a perda do olho, além de possíveis problemas sistêmicos no portador de cavidade anoftálmica (diabetes, hipertensão, colagenoses). Acrescente-se ainda, o fato de que a nossa casuística resulta de cirurgias realizadas por jovens cirurgiões e que estão em treinamento no curso de Residência Médica. Provavelmente, o número de complicações será mais reduzido em mãos experientes. Importante ressaltar que a necrose foi pequena em vários indivíduos, o que não influenciou no resultado final. Assim também a deiscência de sutura, que pode ter boa resolução, observada em alguns de nossos pacientes, não necessitando sistematicamente de reintervenção.

Os granulomas que podem ocorrer nas cavidades que recebem enxerto dermoadiposo podem ser removidos com facilidade. Em geral, os granulomas decorrem de fios de sutura que não são removidos totalmente ou pela persistência de glândulas secretoras no enxerto, inadequadamente removidas quando do preparo do enxerto que deve ter os componentes da epiderme todos removidos. O mesmo pode-se dizer do hirsutismo e de secreção abundante na cavidade que, embora não tenham sido observados em nossos pacientes, podem resultar de preparo inadequado dos enxertos.

A avaliação do quanto e da velocidade da perda de volume nas cavidades tratadas com enxerto dermoadiposo é classicamente reportada e tida como um ponto negativo. No entanto, não há trabalhos científicos randomizados que demonstrem realmente qual é o ritmo de absorção ou de degeneração da gordura implantada. Além disso, a observação deve ser feita a longo prazo. Embora no presente estudo, a avaliação tenha sido feita subjetivamente, pode-se dizer que, com o período de observação mínimo de seis meses, não houve redução significativa do volume do enxerto.

Concluindo, o enxerto dermoadiposo foi aplicado com sucesso na maioria dos indivíduos que compuseram o nosso estudo. Apesar do nosso serviço ser referência na região para tratamento de portadores de cavidade anoftálmica, nossa casuística de pacientes tratados com enxerto dermoadiposo é pequena, demonstrando que mesmo tratando-se de uma técnica simples e eficiente esta ainda é pouco realizada. Portanto,é uma técnica que deve ser ensinada nos serviços de treinamento de jovens cirurgiões, tendo em vista a possibilidade de proporcionar bons resultados para a maioria dos pacientes que dela necessitam.

\section{REFERÊNCIAS}

1. Soares EJC, Dantas RRA, Marback R, Matayoshi S, França VP. Cavidades anoftálmicas. In: Soares EJC, Moura EM, Gonçalves JOR, editores. Cirurgia plástica ocular. São Paulo: Roca; 1997. p. 327.

2. Fernandes JBVD. Cavidade anoftálmica. In: Kara-José N editor. Manual de cirurgia plástica ocular. São Paulo: Roca; 2004. p. 287-8. (Atualidades Oftalmologia USP, 7)

3. Migliori ME, Putterman AM. The domed dermis-fat graft orbital implant. Ophthal Plast Reconstr Surg. 1991;7(1):23-30.

4. Hintschich CR, Beyer-Machule CK. [Dermal fat transplant as autologous orbital implant]. Klin Monbl Augenheilkd. 1996;208(3):135-41. German.

5. Borodic GE, Townsend DJ, Beyer-Machule CK. Dermis fat graft in eviscerated sockets. Ophthal Plast Reconstr Surg. 1989;5(2):144-9.

6. Varene B, Morax S. [Repair of the orbital cavity by a dermoadipose graft after enucleation]. J Fr Ophtalmol. 1986;9(1):45-53. French.

7. Smith B, Petrelli R. Dermis-fat graft as a movable implant within the muscle cone. Am J Ophthalmol. 1978;85(1):62-6.

8. Guberina C, Hornblass A, Metzer MA, Soarez V, Smith B. Autogenous dermis-fat orbital implantation. Arch Ophthalmol. 1983;101(10):1586-90.

9. Hintschich C. [Dermis-fat graft. Possibilities and limitations]. Ophthalmologe. 2003;100(7):518-24. German.

10. Bosniak SL, Nesi F, Smith BC, Schechter B, Cote R. A comparison of motility: autogenous dermis- fat vs synthetic spherical implants. Ophthalmic Surg. 1989;20(12):889-91.

11. Martin PA, Rogers PA, Billson F. Dermis-fat graft: evolution of a living prosthesis. Aust N Z J Ophthalmol. 1986;14(2):161-5.

12. Smith B, Bosniak S, Nesi F, Lisman R. Dermis-fat orbital implantation: 118 cases. Ophthalmic Surg. 1983;14(11):941-3.

13. Bosniak SL. Complications of dermis-fat orbital implantation. Adv Ophthalmic Plast Reconstr Surg. 1990;8:170-81.

14. Bosniak SL. Dermis-fat orbital implantation and complex socket deformities. Adv Ophthalmic Plast Reconstr Surg. 1992;9:131-41.

15. Shore JW, McCord CD Jr, Bergin DJ, Dittmar SJ, Maiorca JP, Burks WR. Management of complications following dermisfat grafting for anophthalmic socket reconstruction. Ophthalmology. 1985;92(10):1342-50.

\section{Endereço para correspondência: \\ Silvia Narikawa \\ Departamento de OFT/ORL/CCP - Faculdade de \\ Medicina de Botucatu - UNESP - \\ CEP 18618-000 - Botucatu (SP), Brasil}

Tel: (14) 38116256

e-mail: silvia.narikawa@hotmail.com 